\title{
Survey for the Assessment and Effect of Different Levels of N- Fertilizers and Spacing on Incidence of False Smut of Rice
}

\author{
M. K. Barnwal ${ }^{1 *}$, A. K. Singh ${ }^{2}$, Arun Kumar ${ }^{3}$ Ravi Kumar $^{3}$ and B. K. Agarwal ${ }^{4}$ \\ ${ }^{1}$ Department of Plant Parhology, ${ }^{2}$ Department of Agronomy, ${ }^{4}$ Department of Soil Science, \\ Birsa Agricultural University, Kanke, Ranchi -834 006, Jharkhand, India \\ ${ }^{3}$ Department of Genetics and Plant Breedimg, Birsa Agricultural University, \\ Ranchi -834 006, Jharkhand, India \\ *Corresponding author
}

\section{A B S T R A C T}

\section{Keywords}

False smut, management, nitrogenous fertilizer, Oryza sativa, spacing, Ustilaginoidea virens

\section{Article Info}

Accepted:

20 September 2020

Available Online:

10 October 2020
Surveys were carried out in eight districts of Jharkhand. Hill infestation, tiller infestation and infected grains with false smut of rice were recorded higher in hybrid rice in comparison to none hybrid (HYV) rice in all surveyed areas of Jharkhand. Hill infestation with false smut of rice ranged from 3.8 to $31.7 \%$ in twenty one rice cultivars. Highest tiller infestation with false smut was recorded in Arize 6444 (24.8\%), followed by PAC 801 (22.5\%), KRH 2 (22.3\%), 25P25 (21.7\%) and PHB 71 (20.3\%). Highest false smut infected grains/panicle was recorded in PAC 801 (8.6) followed by Arize 6444 (8.2). Application of higher level of $\mathrm{N}$-fertilizer in rice recorded higher tiller infestation of false smut of rice, higher number of false smut infected grains /panicle and higher hill infestation of false smut of rice during 2016 and 2017 crop seasons. Closer spacing in rice recorded higher tiller infestation of false smut of rice, higher number of false smut infected grains/ panicle and higher hill infestation of false smut of rice in all three levels $\mathrm{N}$ fertilizers i.e., 90, 120 and $150 \mathrm{Kg} \mathrm{N}^{-1}$ during above two crop seasons. False smut symptom was observed on all three levels of nitrogen having five spacing of rice in both 2014 and 2015 crop seasons. Highest mean grain yield of $78.5 \mathrm{q} \mathrm{ha}^{-1}$ was recorded in plots where N- fertilizer was applied @ $120 \mathrm{Kg} \mathrm{ha}^{-1}$ and spacing of rice was $25 \mathrm{~cm} \mathrm{X} 25 \mathrm{~cm}$, this treatment also recorded mean tiller infestation of $7.8 \%$, lowest mean infected grains/ panicle of 6.3 and lowest mean hill infestation of $11.7 \%$. This treatment was at par with treatment having $\mathrm{N}$ - fertilizers was applied @ $120 \mathrm{Kg} \mathrm{ha}^{-1}$ and spacing of $20 \mathrm{~cm} \mathrm{X} 20$ $\mathrm{cm}$, which recorded mean grain yield of $77.0 \mathrm{q} \mathrm{ha}^{-1}$, mean tiller infestation of $9.1 \%$ and mean hill infestation of $6.6 \%$.

\section{Introduction}

False smut of rice (Oryza sativa L.) caused by Cleviceps oryzae-sativae Hashioka (Imperfect stage - Ustilaginoidea virens
[(Cooke) Takahashi] Hitherto is one of the major production constraints in HYV/ Hybrid rice in all over the world. In India, the disease was called as 'Lakshmi' disease as an indication of bumper harvest. In recent 
decades, the area of hybrid rice cultivation is increasing in India, particularly in Jharkhand State (Pandey and Singh, 2013). In earlier days false smut was considered as a minor disease in rice but now-a-days it is being frequently recorded as a major disease, particularly in hybrid rice growing areas of Jharkhand, causing heavy loss of rice yield both in terms of quality and quantity (Barnwal and Singh, 2011; Barnwal et al., 2016). High incidences of false smut in rice are correlated with increase in many parameters involved in rice production system. These include heavy application of nitrogenous fertilizers, use of responsive cultivars and change in climatic condition etc. associated with modern rice production system (Savary et al., 2004; Barnwal et al., 2018).

HYVs and hybrids are more prone to false smut of rice. Due to this disease of rice, yield loss was recorded up to $49 \%$ in India (Ladhalakshmi, 2012; Kumari and Kumar, 2015). However, differences appear to be from different maturity dates instead of true varietal resistance $(\mathrm{Ou}, 1985)$. At present farmers are applying various level of nitrogenous fertilizer and different spacing in rice cultivation in many districts of Jharkhand State. Disease suppression through optimizing $\mathrm{N}$ - fertilizer and spacing in rice and other crops have been reported by many workers (Long et al., 2000; Riba et al., 2011; Kumar and Yadav, 2012). Therefore, attempt has been made to identify optimum dose of $\mathrm{N}$ - fertilizer and spacing for suppressing false smut disease of rice as an IDM component.

\section{Materials and Methods}

Random survey of rice was conducted at farmers' field of rice growing areas in different villages of eight districts (Ranchi, Lohardaga, Gumla, Khunti, Ramgar, East Singhbhum, West Singhbhum and SaraikelaKharsawan) of Jharkhand during Kharif, 2014 and 2015 to know the false smut incidence of rice. In each district two to five blocks were randomly selected. Three to five villages were surveyed from each block. In each village five to eight farmers' field having one $\mathrm{m}^{2}$ areas each was selected for observation of tiller infestation, false smut infected grains/ panicle and Hill infestation with false smut disease. Ten each smutted and unsmutted (Healthy) panicles were randomly collected from each genotype and grain weight of ten panicles was recorded (Upadhyay and Singh, 2013). The specimens were collected from different locations and collected samples were wrapped in cellophane paper and brought to the laboratory for further studies.

Field trials were conducted during Kharif season, 2016 and 2017 at Darisai research farm of Birsa Agriculture University, Ranchi, Jharkhand.

The trials were laid out in split plot design with three replications. Twenty days old seedlings of susceptible hybrid rice (Arize 6444 Gold) were transplanted @ one seedling per hill in $4^{\text {th }}$ week of July in the main field of the crop.

The plot size was $5.0 \mathrm{~m} \mathrm{X} 4.0 \mathrm{~m}$. The distance between two replications and plot to plot were one meter each. Phosphorus and Potassic $\left(\mathrm{P}_{2} \mathrm{O}_{5}: \mathrm{K}_{2} \mathrm{O}\right)$ fertilizers were applied @ 60:90 $\mathrm{Kg} \mathrm{ha}^{-1}$ each plot. The treatments were three different doses of Nitrogenous $(\mathrm{N})$ fertilizers i.e., 90, 120 and $150 \mathrm{Kg} \mathrm{ha}^{-1}$ were applied treated as a main plots and sub plots of five different spacing i.e., $20 \times 10 \mathrm{~cm}^{2}, 20 \times 15$ $\mathrm{cm}^{2}, 20 \times 20 \mathrm{~cm}^{2}, 25 \times 25 \mathrm{~cm}^{2}$ and $15 \times 15$ $\mathrm{cm}^{2}$ as mentioned in Table 1 . The plots were fertilized with FYM one ton $\mathrm{ha}^{-1}$ and full dose of Phosphatic and potassic fertilizers were applied at the time of transplanting as a basal application. Although $1 / 3^{\text {rd }}$ nitrogenous fertilizer was applied as basal and rest nitrogenous fertilizer was given in two split 
doses i.e., 25 and 50 DAT (Days after transplanting). The necessary agronomic inputs were provided during the crop season. All possible care was taken to prevent insect pest attack by spraying of suitable insecticides according to the necessity. Observations were made as tiller infestation (\%) by dividing the total number of infected tillers to the total number of tillers/ $\mathrm{m}^{2}$ per plot multiplied by 100. Number of infected grains with false smut/panicle were recorded randomly taken 20 smutted panicles per plot at five days before harvesting, whereas, Hill infestation (\%) were observed by cress cross method of 20 hills per plot. Grain yield was also recorded for each plot after harvesting, threshing and eight days sun drying.

\section{Results and Discussion}

Hill infestation, tiller infestation and infected grains with false smut of rice were recorded higher in hybrids in comparison to open pollinated varieties of rice in all surveyed area of Jharkhand. Hill infestation with false smut of rice ranged from 3.8 to 31.7 per cent in twenty one rice varieties /hybrids of eight above surveyed districts of Jharkhand.

Highest tiller infestation with false smut was recorded in Arize 6444 (24.8\%) followed by PAC 801 (22.5\%), KRH 2 (22.3\%), 25P25 $(21.7 \%)$ and PHB $71(20.3 \%)$ whereas, lowest tiller infestation was recorded in IR-64. Highest false smut infected grains/panicle was recorded in PAC 801 (8.6) followed by Arize 6444 (8.2), KRH 2 (7.8), 25P25 (7.3) and 27P52 (7.0). Maximum decrease in weight of ten panicles was observed in Arize 6444 (13.6 g) followed by KRH 2 (10.1 g), PAC 801 $(10.0 \mathrm{~g})$ (Table 1).

Barnwal and Singh (2011) reported the infection of false smut of $68.06 \%$ on HKR and $36 \%$ on PA 6444 . Ladhalakshmi (2012) reported that false smut incidence was very high on hybrids (PA 6444 and PA 6129) in
Haryana, while incidence of disease on PR 114 and PR 116 was higher in Punjab.

\section{N-level}

False smut of rice was observed on all three levels of $\mathrm{N}$ - fertilizer applied in field during both 2016 and 2017 crop seasons. Application of higher level of $\mathrm{N}$-fertilizer in rice recorded higher tiller infestation of false smut of rice, higher number of false smut infected grains /panicle and higher hill infestation of false smut of rice during above two years.

Highest mean grain yield of $73.0 \mathrm{q} \mathrm{ha}^{-1}$ was recorded When nitrogenous fertilizers was applied @ $120 \mathrm{Kg} \mathrm{N} \mathrm{ha}^{-1}$ (F2), this treatment also recorded mean tiller infestation of $10.9 \%$, infected grains per panicle of 8.3 and hill infestation of $16.8 \%$. This treatment was followed by $\mathrm{F} 1$ i.e., $150 \mathrm{Kg} \mathrm{N}$ ha $^{-1}$ which recorded mean grain yield of $70.2 \mathrm{q} \mathrm{ha}^{-1}$, tiller infestation of $13.4 \%$, false smut infected grains/ panicle of 9.7 and hill infestation of 19.9\%. The $\mathrm{F} 3$ treatment $\left(90 \mathrm{Kg} \mathrm{N} \mathrm{ha}{ }^{-1}\right)$ recorded mean grain yield of $55.9 \mathrm{q} \mathrm{ha}^{-1}$, mean tiller infestation of $8.6 \%$, false smut infected grains/ panicle of 6.3 and hill infestation of $14.1 \%$ (Table 2). The present findings corroborates with earlier work of Kabat and Satapathy (2013); Pandey et al., (2008). Long et al., (2000) is also in accordance with our present findings who have reported that the incidence of rice blast increased with increasing levels of nitrogen.

\section{Spacing}

False smut of rice was observed on all five spacing of rice in both 2016 and 2017 crop seasons. Closer spacing in rice recorded higher tiller infestation of false smut of rice, higher number of false smut infected grains /panicle and higher hill infestation of false smut of rice in all three levels $\mathrm{N}$-fertilizers during above two years. 
Table.1 Survey for the assessment of false smut (Ustilaginoidea virens) incidence of different rice cultivars in Jharkhand

\begin{tabular}{|c|c|c|c|c|c|c|}
\hline $\begin{array}{c}\text { Variety/ } \\
\text { Hybrid }\end{array}$ & $\begin{array}{c}\text { *Hill } \\
\text { Infestation } \\
(\boldsymbol{( \% )}\end{array}$ & $\begin{array}{c}* \text { Tiller } \\
\text { infestation } \\
(\mathbf{\%})\end{array}$ & $\begin{array}{c}* \text { No. of Infected } \\
\text { grains/panicle }\end{array}$ & $\begin{array}{c}\text { *Grain weight of 10 } \\
\text { Panicles }\end{array}$ & $\begin{array}{c}\text { Difference } \\
\text { in weight }\end{array}$ \\
\hline Arize 6444 & 31.7 & 24.8 & 8.2 & 46.8 & 32.4 & 14.4 \\
\hline Arize Tej & 22.3 & 18.2 & 6.4 & 41.2 & 32.0 & 9.2 \\
\hline DRRH 2 & 16.0 & 18.3 & 6.8 & 41.2 & 32.3 & 8.9 \\
\hline KRH 2 & 27.3 & 22.3 & 7.8 & 38.8 & 28.7 & 10.1 \\
\hline 27P52 & 26.3 & 20.2 & 7.0 & 39.5 & 30.1 & 9.4 \\
\hline 25P25 & 29.3 & 21.7 & 7.3 & 38.8 & 31.6 & 7.2 \\
\hline PHB 71 & 25.3 & 20.3 & 5.4 & 41.6 & 33.4 & 8.2 \\
\hline 27P31 & 17.0 & 19.7 & 6.3 & 43.2 & 35.6 & 7.6 \\
\hline PAC 801 & 27.7 & 22.5 & 8.6 & 40.8 & 30.8 & 10.0 \\
\hline US 314 & 19.5 & 18.3 & 6.8 & 37.5 & 30.2 & 7.3 \\
\hline Rajendra Mehsuri & 17.7 & 14.0 & 5.7 & 36.8 & 27.8 & 9.0 \\
\hline Birsamati & 18.0 & 12.2 & 5.6 & 32.6 & 24.5 & 6.1 \\
\hline Naveen & 9.7 & 8.7 & 2.5 & 29.8 & 21.7 & 8.1 \\
\hline Swarna & 14.3 & 11.3 & 4.8 & 40.6 & 30.7 & 9.9 \\
\hline Lalat & 6.7 & 4.8 & 3.1 & 37.6 & 29.8 & 7.8 \\
\hline Abhisek & 5.3 & 3.8 & 1.8 & 35.4 & 29.2 & 6.2 \\
\hline MTU 1010 & 8.7 & 7.2 & 2.2 & 37.9 & 32.2 & 5.7 \\
\hline IR 64 & 3.8 & 3.2 & 1.7 & 39.8 & 31.6 & 8.2 \\
\hline MTU 1001 & 12.7 & 6.7 & 3.3 & 36.7 & 32.6 & 6.1 \\
\hline Sahbhagi & 5.6 & 3.1 & 2.3 & 38.5 & 31.3 & 7.2 \\
\hline BVD 110 & 4.8 & 3.6 & 1.9 & 37.6 & 31.2 & 6.4 \\
\hline Mean of two years data $(\mathbf{2 0 1 4}$ and 2015) & & & & & \\
\hline
\end{tabular}


Table.2 Evaluation of different levels of nitrogenous fertilizer on incidence of false smut disease and grain yield of rice

\begin{tabular}{|c|c|c|c|c|c|c|c|c|c|c|c|c|}
\hline \multirow[t]{2}{*}{$\begin{array}{l}\text { Treatments } \\
\text { (N- Fertilizer) }\end{array}$} & \multicolumn{3}{|c|}{ *Tiller infestation (\%) } & \multicolumn{3}{|c|}{$\begin{array}{c}\text { *Number of infected } \\
\text { grains/panicle }\end{array}$} & \multicolumn{3}{|c|}{$\begin{array}{l}\text { *Hill } \\
\text { infestation }(\%)\end{array}$} & \multicolumn{3}{|c|}{ *Grain yield (q/ha) } \\
\hline & 2016 & 2017 & Mean & 2016 & 2017 & Mean & 2016 & 2017 & Mean & 2016 & 2017 & Mean \\
\hline F1 & $\begin{array}{c}12.9 \\
(20.5)\end{array}$ & $\begin{array}{c}13.8 \\
(21.6)\end{array}$ & $\begin{array}{c}13.4 \\
(21.1)\end{array}$ & 8.5 & 10.9 & 9.7 & $\begin{array}{c}22.3 \\
(27.7)\end{array}$ & $\begin{array}{c}17.4 \\
(24.0)\end{array}$ & $\begin{array}{c}19.9 \\
(25.9)\end{array}$ & 69.2 & 71.1 & 70.2 \\
\hline F2 & $\begin{array}{c}10.2 \\
(18.4)\end{array}$ & $\begin{array}{c}11.6 \\
(19.8)\end{array}$ & $\begin{array}{c}10.9 \\
(19.1)\end{array}$ & 7.5 & 9.1 & 8.3 & $\begin{array}{c}18.9 \\
(25.5)\end{array}$ & $\begin{array}{c}14.7 \\
(22.3)\end{array}$ & $\begin{array}{c}16.8 \\
(23.9)\end{array}$ & 72.3 & 73.7 & 73.0 \\
\hline F3 & $\begin{array}{c}8.2 \\
(16.6)\end{array}$ & $\begin{array}{c}9.0 \\
(17.6)\end{array}$ & $\begin{array}{c}8.6 \\
(17.1)\end{array}$ & 5.8 & 6.7 & 6.3 & $\begin{array}{l}15.9 \\
(23.1)\end{array}$ & $\begin{array}{l}12.3 \\
(20.1)\end{array}$ & $\begin{array}{l}14.1 \\
(21.6)\end{array}$ & 56.8 & 55.1 & 55.9 \\
\hline $\begin{array}{l}\text { CD at } 5 \% \\
\text { CV }(\%)\end{array}$ & $\begin{array}{c}2.1 \\
19.7\end{array}$ & $\begin{array}{c}2.80 \\
11.8\end{array}$ & $\begin{array}{c}2.6 \\
16.3\end{array}$ & NS & $\begin{array}{c}2.81 \\
17.60\end{array}$ & NS & NS & $\begin{array}{r}2.42 \\
17.50\end{array}$ & NS & $\begin{array}{c}5.78 \\
17.60\end{array}$ & $\begin{array}{c}3.40 \\
15.10\end{array}$ & $\begin{array}{l}4.82 \\
16.5\end{array}$ \\
\hline
\end{tabular}

Table.4.1 Evaluation of different levels of nitrogenous fertilizer and spacing on incidence of false smut of rice

\begin{tabular}{|c|c|c|c|c|c|c|c|c|c|c|c|c|}
\hline \multirow[t]{2}{*}{$\begin{array}{l}\text { Treatments } \\
\text { (Spacing) }\end{array}$} & \multicolumn{3}{|c|}{ *Tiller infestation (\%) } & \multicolumn{3}{|c|}{$\begin{array}{c}* \text { Number of infected } \\
\text { grains/panicle }\end{array}$} & \multicolumn{3}{|c|}{$*$ Hill infestation (\%) } & \multicolumn{3}{|c|}{ *Grain yield (q/ha) } \\
\hline & 2016 & 2017 & Mean & 2016 & 2017 & Mean & 2016 & 2017 & Mean & 2016 & 2017 & Mean \\
\hline S1 & $\begin{array}{c}13.9 \\
(21.6)\end{array}$ & $\begin{array}{c}10.7 \\
(18.9)\end{array}$ & $\begin{array}{c}12.3 \\
(20.3)\end{array}$ & 8.6 & 11.8 & 10.4 & $\begin{array}{c}24.6 \\
(29.0)\end{array}$ & $\begin{array}{c}18.9 \\
(25.6)\end{array}$ & $\begin{array}{c}21.8 \\
(27.3)\end{array}$ & 61.3 & 60.7 & 61.0 \\
\hline $\mathbf{S 2}$ & $\begin{array}{c}10.6 \\
(18.8)\end{array}$ & $\begin{array}{c}9.0 \\
(17.4)\end{array}$ & $\begin{array}{c}9.8 \\
(18.1)\end{array}$ & 8.0 & 9.5 & 8.8 & $\begin{array}{c}20.0 \\
(26.0)\end{array}$ & $\begin{array}{c}16.1 \\
(23.5)\end{array}$ & $\begin{array}{c}18.1 \\
(24.8)\end{array}$ & 65.3 & 66.9 & 66.6 \\
\hline $\mathbf{S 3}$ & $\begin{array}{c}7.8 \\
(16.1)\end{array}$ & $\begin{array}{c}8.3 \\
(16.7)\end{array}$ & $\begin{array}{c}8.1 \\
(16.4)\end{array}$ & 6.3 & 7.1 & 6.7 & $\begin{array}{l}17.2 \\
(24.4)\end{array}$ & $\begin{array}{c}11.2 \\
(19.1)\end{array}$ & $\begin{array}{c}14.2 \\
(12.8)\end{array}$ & 69.3 & 70.0 & 69.7 \\
\hline S4 & $\begin{array}{c}6.7 \\
(14.8)\end{array}$ & $\begin{array}{c}4.5 \\
(14.8)\end{array}$ & $\begin{array}{c}5.6 \\
(14.8)\end{array}$ & 4.4 & 6.5 & 5.5 & $\begin{array}{c}11.6 \\
(19.6)\end{array}$ & $\begin{array}{c}10.5 \\
(18.2)\end{array}$ & $\begin{array}{c}11.1 \\
(18.9)\end{array}$ & 73.9 & 72.4 & 73.2 \\
\hline S5 & $\begin{array}{l}12.8 \\
(20.7)\end{array}$ & $\begin{array}{c}12.3 \\
(20.4)\end{array}$ & $\begin{array}{c}12.6 \\
(20.6)\end{array}$ & 8.9 & 9.4 & 9.2 & $\begin{array}{c}21.6 \\
(27.3)\end{array}$ & $\begin{array}{c}17.2 \\
(24.3)\end{array}$ & $\begin{array}{c}19.4 \\
(25.8)\end{array}$ & 60.7 & 63.2 & 62.0 \\
\hline $\begin{array}{l}\text { CD at 5\% } \\
\text { CV }(\%)\end{array}$ & $\begin{array}{c}3.4 \\
18.6\end{array}$ & $\begin{array}{c}2.3 \\
14.1\end{array}$ & $\begin{array}{c}3.1 \\
16.6\end{array}$ & NS & $\begin{array}{c}1.8 \\
24.1\end{array}$ & NS & $\begin{array}{l}4.79 \\
12.9\end{array}$ & $\begin{array}{c}3.8 \\
12.9\end{array}$ & $\begin{array}{c}4.5 \\
12.9\end{array}$ & $\begin{array}{c}6.8 \\
11.0\end{array}$ & $\begin{array}{c}7.2 \\
15.1\end{array}$ & $\begin{array}{c}7.1 \\
14.3\end{array}$ \\
\hline
\end{tabular}


Table.4.2 Evaluation of different levels of nitrogenous fertilizer and spacing on incidence of false smut of rice

\begin{tabular}{|c|c|c|c|c|c|c|c|c|c|c|c|c|}
\hline \multirow{3}{*}{$\mathbf{F i}$} & \multicolumn{12}{|c|}{ *Tiller infestation (\%) } \\
\hline & \multicolumn{3}{|c|}{ F1 } & \multicolumn{3}{|c|}{ F2 } & \multicolumn{3}{|c|}{ F3 } & \multicolumn{3}{|c|}{ Mean } \\
\hline & 2016 & 2017 & Mean & 2016 & 2017 & Mean & 2016 & 2017 & Mean & 2016 & 2017 & Mean \\
\hline S1 & $\begin{array}{c}19.7 \\
(26.1)\end{array}$ & $\begin{array}{c}19.7 \\
(26.1)\end{array}$ & $\begin{array}{c}19.7 \\
(26.1)\end{array}$ & $\begin{array}{c}11.6 \\
(19.9)\end{array}$ & $\begin{array}{c}14.7 \\
(22.5)\end{array}$ & $\begin{array}{c}13.0 \\
(21.2)\end{array}$ & $\begin{array}{c}10.3 \\
(18.8)\end{array}$ & $\begin{array}{c}10.7 \\
(18.9)\end{array}$ & $\begin{array}{c}10.5 \\
(18.9)\end{array}$ & $\begin{array}{c}13.9 \\
(21.6)\end{array}$ & $\begin{array}{c}15.0 \\
(22.5)\end{array}$ & $\begin{array}{c}14.5 \\
(22.1)\end{array}$ \\
\hline $\mathbf{S 2}$ & $\begin{array}{c}12.3 \\
(20.2)\end{array}$ & $\begin{array}{c}13.0 \\
(21.1)\end{array}$ & $\begin{array}{c}12.4 \\
(20.7)\end{array}$ & $\begin{array}{c}10.7 \\
(18.9)\end{array}$ & $\begin{array}{c}11.0 \\
(19.2)\end{array}$ & $\begin{array}{c}10.9 \\
(19.1)\end{array}$ & $\begin{array}{c}8.8 \\
(17.3)\end{array}$ & $\begin{array}{c}9.0 \\
(17.4)\end{array}$ & $\begin{array}{c}8.9 \\
(17.3)\end{array}$ & $\begin{array}{c}10.6 \\
(18.8)\end{array}$ & $\begin{array}{c}11.0 \\
(19.2)\end{array}$ & $\begin{array}{c}10.8 \\
(19.0)\end{array}$ \\
\hline S3 & $\begin{array}{c}9.0 \\
(17.3)\end{array}$ & $\begin{array}{c}11.0 \\
(19.3)\end{array}$ & $\begin{array}{c}10.0 \\
(18.3)\end{array}$ & $\begin{array}{c}7.8 \\
(16.1)\end{array}$ & $\begin{array}{c}10.3 \\
(18.7)\end{array}$ & $\begin{array}{c}9.1 \\
(17.4)\end{array}$ & $\begin{array}{c}6.6 \\
(14.9)\end{array}$ & $\begin{array}{c}8.3 \\
(16.7)\end{array}$ & $\begin{array}{c}7.5 \\
(15.8)\end{array}$ & $\begin{array}{c}7.8 \\
(16.1)\end{array}$ & $\begin{array}{c}9.9 \\
(18.2)\end{array}$ & $\begin{array}{c}8.9 \\
(17.7)\end{array}$ \\
\hline S4 & $\begin{array}{c}8.3 \\
(16.5)\end{array}$ & $\begin{array}{c}10.3 \\
(18.6)\end{array}$ & $\begin{array}{c}9.3 \\
(17.6)\end{array}$ & $\begin{array}{c}7.3 \\
(15.5)\end{array}$ & $\begin{array}{c}8.3 \\
(16.7)\end{array}$ & $\begin{array}{c}7.8 \\
(16.1)\end{array}$ & $\begin{array}{c}4.5 \\
(12.3)\end{array}$ & $\begin{array}{c}4.5 \\
(14.8)\end{array}$ & $\begin{array}{c}4.5 \\
(13.6)\end{array}$ & $\begin{array}{c}6.7 \\
(14.8)\end{array}$ & $\begin{array}{c}7.7 \\
(16.7)\end{array}$ & $\begin{array}{c}7.2 \\
(15.8)\end{array}$ \\
\hline S5 & $\begin{array}{c}15.0 \\
(22.4)\end{array}$ & $\begin{array}{c}15.0 \\
(22.4)\end{array}$ & $\begin{array}{c}15.0 \\
(22.4)\end{array}$ & $\begin{array}{c}12.7 \\
(20.7)\end{array}$ & $\begin{array}{c}13.7 \\
(21.7)\end{array}$ & $\begin{array}{c}13.2 \\
(21.2)\end{array}$ & $\begin{array}{c}10.7 \\
(19.0)\end{array}$ & $\begin{array}{c}12.3 \\
(20.4)\end{array}$ & $\begin{array}{c}11.5 \\
(19.7)\end{array}$ & $\begin{array}{c}12.8 \\
(20.7)\end{array}$ & $\begin{array}{c}13.7 \\
(21.6)\end{array}$ & $\begin{array}{c}13.3 \\
(21.2)\end{array}$ \\
\hline Mean & $\begin{array}{c}12.9 \\
(20.5)\end{array}$ & $\begin{array}{c}13.8 \\
(21.6)\end{array}$ & $\begin{array}{c}13.4 \\
(21.1)\end{array}$ & $\begin{array}{c}10.2 \\
(18.4)\end{array}$ & $\begin{array}{c}11.6 \\
(19.8)\end{array}$ & $\begin{array}{c}10.9 \\
(19.1)\end{array}$ & $\begin{array}{c}8.2 \\
(16.4)\end{array}$ & $\begin{array}{c}9.0 \\
(17.6)\end{array}$ & $\begin{array}{c}17.1 \\
(17.0)\end{array}$ & & & \\
\hline & & & & 2015 & 2016 & Mean & & & & & & \\
\hline $\mathrm{CV}(\%)$ & & & $\begin{array}{l}F_{1}-F_{3-} \\
S_{1}-S_{5-} \\
F_{1} S_{1}-F_{1} S_{5^{-}} \\
F_{1} S_{1}-F_{3} S_{5^{-}} \\
\text {CV1- } \\
\text { CV2- }\end{array}$ & $\begin{array}{c}2.10 \\
3.44 \\
\mathrm{NS} \\
\mathrm{NS} \\
19.7 \\
18.6\end{array}$ & $\begin{array}{c}2.80 \\
2.30 \\
\mathrm{NS} \\
\mathrm{NS} \\
11.8 \\
14.1\end{array}$ & $\begin{array}{c}2.63 \\
3.10 \\
\mathrm{NS} \\
\mathrm{NS} \\
16.3 \\
16.6\end{array}$ & & & & & & \\
\hline $\begin{array}{l}\text { Where F1- } \\
\text { X15cm } \\
\text { *Mean of th }\end{array}$ & $\begin{array}{l}0 \mathrm{Kg} \mathrm{N} \text { ha } \\
\text { ee replicat }\end{array}$ & & $\begin{array}{l}\mathbf{N} \text { ha- }{ }^{1}, \mathbf{F 3 - 9 0} \\
\text { ures in parenth }\end{array}$ & $0 \mathrm{Kg} \mathrm{N} \mathrm{h}$ & $\begin{array}{l}\text { S1- } 20 \mathrm{cn} \\
\text { nsformed }\end{array}$ & $10 \mathrm{~cm}, \mathrm{~S} 2-$ & $0 \mathrm{~cm} \times 15$ & S3- 20 & $X 20 \mathrm{~cm}$ & $25 \mathrm{~cm}$ & cm anc & $-15 \mathrm{~cm}$ \\
\hline
\end{tabular}


Table.4.3 Evaluation of different levels of nitrogenous fertilizer and spacing on incidence of false smut of rice

\begin{tabular}{|c|c|c|c|c|c|c|c|c|c|c|c|c|}
\hline \multirow[t]{3}{*}{$\mathbf{F i}$} & \multicolumn{12}{|c|}{ * Number of infected grains/panicle } \\
\hline & \multicolumn{3}{|c|}{ F1 } & \multicolumn{3}{|c|}{ F2 } & \multicolumn{3}{|c|}{ F3 } & \multicolumn{3}{|c|}{ Mean } \\
\hline & 2016 & 2017 & Mean & 2016 & 20167 & Mean & 2016 & 2017 & Mean & 2016 & 2017 & Mean \\
\hline S1 & 10.3 & 16.2 & 13.3 & 9.2 & 10.8 & 10.0 & 6.3 & 8.4 & 7.4 & 8.6 & 11.8 & 10.2 \\
\hline S2 & 8.7 & 11.9 & 10.3 & 7.8 & 10.1 & 9.0 & 7.5 & 6.5 & 7.0 & 8.0 & 9.5 & 8.8 \\
\hline S3 & 8.3 & 8.3 & 8.3 & 6.0 & 7.1 & 6.6 & 5.3 & 5.9 & 5.6 & 6.3 & 7.1 & 6.6 \\
\hline S4 & 5.1 & 7.6 & 6.4 & 5.7 & 6.8 & 6.3 & 4.7 & 5.2 & 5.0 & 4.4 & 6.5 & 5.9 \\
\hline S5 & 9.8 & 10.3 & 10.1 & 8.7 & 10.3 & 9.5 & 2.3 & 7.6 & 5.0 & 8.9 & 9.4 & 9.2 \\
\hline Mean & 8.5 & 10.9 & 9.7 & 7.5 & 9.1 & 8.3 & 5.8 & 6.7 & 6.3 & 7.24 & 8.86 & 8.14 \\
\hline & & & & 2015 & 2016 & Mean & & & & & & \\
\hline $\begin{array}{l}\text { CD at } 5 \% \\
\text { CV }(\%)\end{array}$ & $\begin{array}{l}\mathrm{F}_{1^{-}} \mathrm{F}_{3^{-}} \\
\mathrm{S}_{1^{-}} \mathrm{S}_{5^{-}} \\
\mathrm{F}_{1} \mathrm{~S}_{1^{-}} \mathrm{F}_{1} \mathrm{~S}_{5^{-}} \\
\mathrm{F}_{1} \mathrm{~S}_{1}-\mathrm{F}_{3} \mathrm{~S}_{5} \\
\mathrm{CV} 1^{-} \\
\mathrm{CV} 2^{-}\end{array}$ & & & $\begin{array}{l}\text { NS } \\
\text { NS } \\
\text { NS } \\
\text { NS }\end{array}$ & $\begin{array}{c}2.8 \\
1.8 \\
3.1 \\
3.9 \\
17.6 \\
24.1\end{array}$ & $\begin{array}{l}\text { NS } \\
\text { NS } \\
\text { NS } \\
\text { NS }\end{array}$ & & & & & & \\
\hline $\begin{array}{l}\text { Where F1- } \\
\text { X15cm } \\
\text { * Mean of th }\end{array}$ & $\begin{array}{l}150 \mathrm{Kg} \mathrm{N} \text { ha- } \\
\text { ree replicatio }\end{array}$ & $\begin{array}{r}2-120 \\
F\end{array}$ & $s$ in par & ses are & $\begin{array}{l}51-20 \mathrm{~cm} \\
\text { formed } \mathrm{i}\end{array}$ & $\begin{array}{l}\mathrm{X} 10 \mathrm{~cm}, \mathrm{S2} \\
0 \text { arc sine }\end{array}$ & & S3- 20 & & & & \\
\hline
\end{tabular}


Table.4.4 Evaluation of different levels of nitrogenous fertilizer and spacing on grain yield of rice

\begin{tabular}{|c|c|c|c|c|c|c|c|c|c|c|c|c|}
\hline \multirow{3}{*}{$\begin{array}{l}\text { Fi } \\
\text { Si }\end{array}$} & \multicolumn{12}{|c|}{$*$ Hill infestation (\%) } \\
\hline & \multicolumn{3}{|c|}{ F1 } & \multicolumn{3}{|c|}{ F2 } & \multicolumn{3}{|c|}{ F3 } & \multicolumn{3}{|c|}{ Mean } \\
\hline & 2016 & 2017 & Mean & 2016 & 2017 & Mean & 2016 & 2017 & Mean & 2016 & 2017 & Mean \\
\hline S1 & $\begin{array}{c}28.3 \\
(31.1)\end{array}$ & $\begin{array}{c}21.6 \\
(27.6)\end{array}$ & $\begin{array}{c}25.5 \\
(29.4)\end{array}$ & $\begin{array}{c}23.7 \\
(28.7)\end{array}$ & $\begin{array}{c}18.3 \\
(25.0)\end{array}$ & $\begin{array}{c}16.0 \\
(26.9)\end{array}$ & $\begin{array}{c}21.7 \\
(27.2)\end{array}$ & $\begin{array}{c}16.7 \\
(24.1)\end{array}$ & $\begin{array}{c}19.2 \\
(25.2)\end{array}$ & $\begin{array}{c}24.6 \\
(29.0)\end{array}$ & $\begin{array}{c}18.9 \\
(25.6)\end{array}$ & $\begin{array}{c}21.8 \\
(27.3)\end{array}$ \\
\hline S2 & $\begin{array}{c}21.7 \\
(27.5)\end{array}$ & $\begin{array}{c}18.3 \\
(25.0)\end{array}$ & $\begin{array}{c}20.0 \\
(26.3)\end{array}$ & $\begin{array}{c}18.3 \\
(25.0)\end{array}$ & $\begin{array}{c}15.0 \\
(22.6)\end{array}$ & $\begin{array}{c}16.7 \\
(23.8)\end{array}$ & $\begin{array}{c}20.0 \\
(25.3)\end{array}$ & $\begin{array}{c}15.0 \\
(22.6)\end{array}$ & $\begin{array}{c}17.5 \\
(24.0)\end{array}$ & $\begin{array}{c}20.0 \\
(26.0)\end{array}$ & $\begin{array}{c}16.1 \\
(23.5)\end{array}$ & $\begin{array}{c}18.1 \\
(24.8)\end{array}$ \\
\hline S3 & $\begin{array}{c}20.0 \\
(26.5)\end{array}$ & $\begin{array}{c}13.7 \\
(21.3)\end{array}$ & $\begin{array}{c}16.4 \\
(23.9)\end{array}$ & $\begin{array}{c}16.7 \\
(24.1)\end{array}$ & $\begin{array}{c}11.7 \\
(19.9)\end{array}$ & $\begin{array}{c}14.2 \\
(22.0)\end{array}$ & $\begin{array}{c}15.0 \\
(22.6)\end{array}$ & $\begin{array}{c}8.3 \\
(16.2)\end{array}$ & $\begin{array}{c}11.7 \\
(19.4)\end{array}$ & $\begin{array}{c}17.2 \\
(24.4)\end{array}$ & $\begin{array}{c}11.2 \\
(19.1)\end{array}$ & $\begin{array}{c}14.2 \\
(21.8)\end{array}$ \\
\hline S4 & $\begin{array}{c}15.0 \\
(22.6)\end{array}$ & $\begin{array}{c}13.3 \\
(19.9)\end{array}$ & $\begin{array}{c}14.2 \\
(16.3)\end{array}$ & $\begin{array}{c}11.7 \\
(19.9)\end{array}$ & $\begin{array}{c}11.7 \\
(19.9)\end{array}$ & $\begin{array}{c}11.7 \\
(19.9)\end{array}$ & $\begin{array}{c}8.3 \\
(16.2)\end{array}$ & $\begin{array}{c}6.7 \\
(14.8)\end{array}$ & $\begin{array}{c}7.5 \\
(15.5)\end{array}$ & $\begin{array}{c}11.6 \\
(19.6)\end{array}$ & $\begin{array}{c}10.5 \\
(18.2)\end{array}$ & $\begin{array}{c}11.1 \\
(18.9)\end{array}$ \\
\hline S5 & $\begin{array}{c}26.7 \\
(30.9)\end{array}$ & $\begin{array}{c}20.0 \\
(26.1)\end{array}$ & $\begin{array}{c}23.4 \\
(28.5)\end{array}$ & $\begin{array}{c}25.0 \\
(29.7)\end{array}$ & $\begin{array}{c}16.7 \\
(24.1)\end{array}$ & $\begin{array}{c}20.9 \\
(26.9)\end{array}$ & $\begin{array}{c}13.3 \\
(21.1)\end{array}$ & $\begin{array}{c}15.0 \\
(22.6)\end{array}$ & $\begin{array}{c}14.2 \\
(21.9)\end{array}$ & $\begin{array}{c}21.6 \\
(27.3)\end{array}$ & $\begin{array}{c}17.2 \\
(24.3)\end{array}$ & $\begin{array}{c}19.4 \\
(25.8)\end{array}$ \\
\hline Mean & $\begin{array}{c}22.3 \\
(27.7)\end{array}$ & $\begin{array}{c}17.4 \\
(24.0)\end{array}$ & $\begin{array}{c}19.9 \\
(25.9)\end{array}$ & $\begin{array}{c}18.9 \\
(25.5)\end{array}$ & $\begin{array}{c}14.7 \\
(22.3)\end{array}$ & $\begin{array}{c}16.8 \\
(23.9)\end{array}$ & $\begin{array}{c}15.9 \\
(23.1)\end{array}$ & $\begin{array}{c}12.3 \\
(20.1)\end{array}$ & $\begin{array}{c}14.0 \\
(21.2)\end{array}$ & $\begin{array}{c}19.0 \\
(25.6)\end{array}$ & $\begin{array}{c}14.8 \\
(22.1)\end{array}$ & $\begin{array}{l}16.9 \\
(23.7)\end{array}$ \\
\hline & & & & 2015 & 2016 & Mean & & & & & & \\
\hline $\mathrm{CV}(\%)$ & $\begin{array}{l}\mathrm{F}_{1^{-}} \mathrm{F}_{3^{-}} \\
\mathrm{S}_{1^{-}} \mathrm{S}_{5^{-}} \\
\mathrm{F}_{1} \mathrm{~S}_{1^{-}} \mathrm{F}_{1} \mathrm{~S}_{5} \\
\mathrm{~F}_{1} \mathrm{~S}_{1}-\mathrm{F}_{3} \mathrm{~S} \\
\mathrm{CV} 1- \\
\mathrm{CV} 2^{-}\end{array}$ & & & $\begin{array}{l}\text { NS } \\
\text { NS } \\
\text { NS } \\
\text { NS }\end{array}$ & $\begin{array}{l}2.42 \\
3.80 \\
\mathrm{NS} \\
\mathrm{NS} \\
17.5 \\
12.9\end{array}$ & $\begin{array}{c}\text { NS } \\
\text { NS } \\
\text { NS } \\
\text { NS }\end{array}$ & & & & & & \\
\hline $\begin{array}{l}\text { Where F1 } \\
\text { X15cm } \\
* \text { Mean of } t\end{array}$ & $\begin{array}{l}150 \mathrm{Kg} \mathrm{N} \text { ha } \\
\text { aree replicati }\end{array}$ & ${ }^{1} \mathrm{~F} 2-12$ & ures in $\mathrm{p}$ & $90 \mathrm{Kg} \mathrm{N}$ & S1-20c & $10 \mathrm{~cm}$, & $\begin{array}{l}20 \mathrm{~cm} \mathrm{X} \\
\text { values }\end{array}$ & $\mathrm{m}, \mathrm{S3-2}$ & $X 20 \mathrm{~cm}$ & $4-25 \mathrm{~cm}$ & $5 \mathrm{~cm}$ and & $-15 \mathrm{~cm}$ \\
\hline
\end{tabular}


Fig.1 Map showing survey assessment of false smut (Ustilaginoidea virens) incidence of different rice cultivars in different districts of Jharkhand
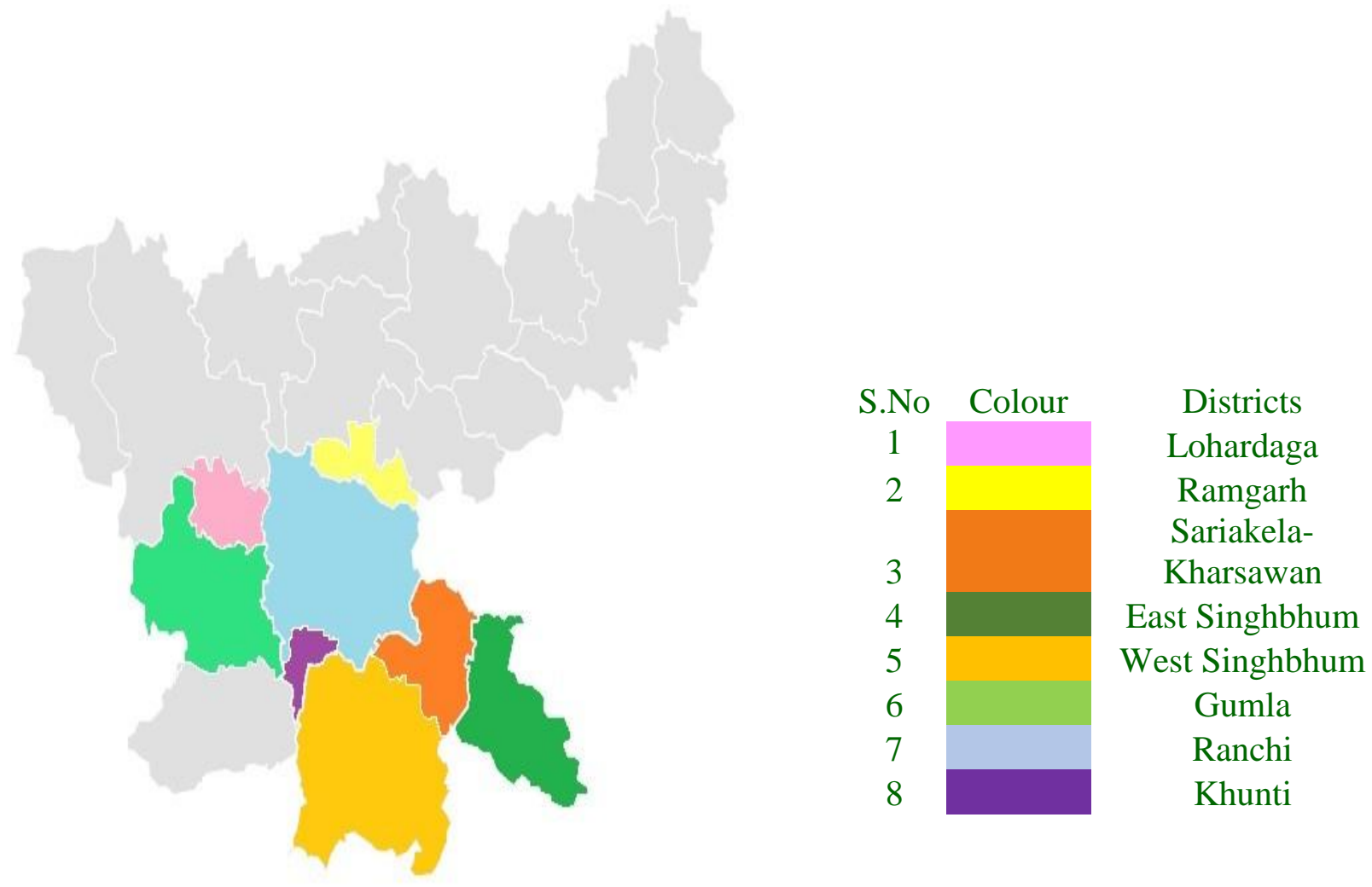

Highest mean grain yield of $73.2 \mathrm{q} \mathrm{ha}^{-1}$ was recorded in plots having spacing of rice was $25 \mathrm{~cm} \times 25 \mathrm{~cm}(\mathrm{~S} 4)$, this treatment also recorded lowest mean tiller infestation of $5.6 \%$, lowest mean infected grains/ panicle of 5.5 and lowest mean hill infestation of $11.1 \%$. This treatment was at par with S3 i.e., spacing of $20 \times 20 \mathrm{~cm}$, which recorded mean grain yield of $69.7 \mathrm{q} \mathrm{ha}^{-1}$, mean tiller infestation of $8.1 \%$, mean infected grains/ panicle of 6.7 and mean hill infestation of $14.2 \%$. The next best treatment in order of superiority was is $\mathrm{S} 2(20 \mathrm{~cm} \mathrm{X} 15 \mathrm{~cm})$ recorded mean grain yield of $66.6 \mathrm{q} \mathrm{ha}^{-1}$, mean tiller infestation of $9.8 \%$, infected grains per panicle of 8.8 and hill infestation of $18.1 \%$ (Table 3). Pathak et al., (2013) has also been observed that the incidence of false smut was significantly lower in SRI system $(25 \mathrm{~cm} \mathrm{X} 25 \mathrm{~cm})$ of rice cultivation in comparison to traditional system $(20 \mathrm{~cm}$ X $10 \mathrm{~cm})$ of rice cultivation. Grain and straw yields were also higher SRI in comparison to traditional system of rice cultivation. Riba et al., (2011) who also reported higher incidence of fungal diseases in traditional system of rice cultivation in comparison to SRI method of rice cultivation in North Eastern region

\section{Nitrogen X Spacing of rice}

Symptom of false smut was observed on all three doses of nitrogen having five spacing of rice in both 2016 and 2017 crop seasons. Highest mean grain yield of $78.5 \mathrm{q} \mathrm{ha}^{-1}$ was recorded in plots where $\mathrm{N}$-fertilizer was 
applied @120 Kg ha ${ }^{-1}$ and Spacing of rice was $25 \mathrm{~cm} \mathrm{X} 25 \mathrm{~cm}$ (S4), this treatment also recorded mean tiller infestation of $7.8 \%$, lowest mean infected grains per panicle of 6.3 and lowest mean hill infestation of $11.7 \%$. This treatment was at par with treatment having $\mathrm{N}$ - fertilizer was applied @ $120 \mathrm{Kg} \mathrm{ha}$ 1 and spacing of $20 \mathrm{~cm} \times 20 \mathrm{~cm}$, which recorded mean grain yield of 77.0 $\mathrm{q} \mathrm{ha}{ }^{-1}$, mean tiller infestation of $9.1 \%$, mean infected grains per panicle of 6.6 and mean hill infestation of $14.2 \%$. The next best treatment in order of superiority was is F1S4 i.e., N-fertilizer was applied @ $150 \mathrm{Kg} \mathrm{ha}^{-1}$ (F1) and spacing of rice was $25 \mathrm{~cm} \mathrm{X} 25 \mathrm{~cm}$ (S4) which recorded mean grain yield of 76.2 $\mathrm{q} \mathrm{ha}^{-1}$, mean tiller infestation of $9.3 \%$, false smut infected grains per panicle of 6.4 and hill infestation of $14.2 \%$ (Table 4.1- 4.4). Similar findings have been recorded by various workers against rice and other crops (Long et al., 2000; Riba et al., 2011; Kumar and Yadav, 2012).

\section{References}

Barnwal, M.K. and Singh, R. (2011). Annual progress report, Directorate of Rice Research (ICAR), Rajendranagar, Hyderabad. pp. 100-105.

Barnwal M.K., Sah, A., Singh D.N. and Agarwal, B.K. and Sharma, R.B. (2012). Chemical management of false smut of hybrid rice (Oryza sativa L.) under rainfed lowland ecosystem J. Res. (BAU). 24(1): 59-62.

Barnwal, M. K., Roy, P. and Kumar, T.R. (2016). Evaluation of fungicides against false smut of rice. Indian Phytopath. 69(4S): 522-524.

Barnwal, M. K., Singh, D.N., Barnwal, S.K. and Agarwal, B. K. (2018). Evaluation of rice hybrids for resistance to false smut and yield of rice. Journal of Pharmacogony and Phytochemistry Spl.: 1689-169.
Kabat, B. and Satapathy, M.R. (2013). Effect of planting dates and $\mathrm{N}$ levels on grain yield and $\mathrm{N}$ uptake by hybrid rice, Oryza 50(4): 409-411.

Kumar, B. and Yadav, R. (2012). Influence of nitrogen fertilizer dose on blast of finger millet caused by Pyicularia grisea Indian Phytopath. 65(1): 52-55.

Kumari, S. and Kumar J. (2015). Evaluation of yield losses and management practices of false smut of rice (Oryza sativa). Indian Phytopath. 68(1): 45-49.

Ladhalakshimi, D., Laha, G.S., Singh Ram, Krishnaveni, D., Prasad M.S., Mangrauthia S.K., Prakasam, V., Yugander, A. and Virakatmath B.C. (2012). False smut, a threatening disease of rice. Directorate of Rice Research, Rajendranagar, Hyderabad, pp 31.

Long, D.H., Lee F.N. and Tebeest, D.O. (2000). Effect of nitrogen fertilization on disease progress of rice blast on susceptible and resistant cultivars. Plant Dis. 84: 403-409.

Ou, S.H. (1985). Rice Diseases, $2^{\text {nd }}$ edn. Commonwealth Mycological Institute (Commonwealth Agricultural Bureaux), Kew, Survey, UK, pp.307-311.

Pathak, M. Shakywar, R.C. and Sah, D. (2013). Biodiversity of insect pests, natural enemies and diseases in SRI and traditional system of rice cultivation. In North East region of India. Oryza 50(4): 370-374.

Pandey, M.P. and Singh, D.N. (2013). Current status and future prospects of hybrid rice in Jharkhand, India. International Conference of Rice at IRRI, Philippines held on Oct., 2013.

Pandey, N. Verma, A.K. and Tripathy, R.S. (2008). Effect of planting dates and Nitrogen levels of $\mathrm{N}$ concentration in the leaf, grain yield and $\mathrm{N}$-uptake by hybrid rice. Oryza 50(4): 370-374.

Riba, T., Pathak, Mahesh and Shakywar 
(2011). Insect pest diseases in rainfed rice ecosystem of East Siang district, Arunachal Pradesh, Tripura Science Congress held at Agartala, Sep., 8-9, 2011, pp 35.

Savary, S., Mew, T.W., Leung, H., Cruz, C.M.V. and Leach, J.E. (2004). Looking Ahead in Rice Disease Research and Management. Critical Reviews in Plant Sci. 23: 103-127.
Singh, S. Lal, A. A. Simon, S. Singh, A., Yaduman, R., Kamaluddeen and David, A. (2014). Survey of false smut (Ustlilaginoidea virens) in selected districts of Uttar Pradesh, India. The Bioscan 9(1): 389-392.

Upadhyay, A.L., and Singh, R.V. (2013). Yield loss assessment in rice due to false smut. Ann. Plant Soil Res. 15: $173-174$.

\section{How to cite this article:}

Barnwal, M. K., A. K. Singh, Arun Kumar, Ravi Kumar and Agarwal, B. K. 2020. Survey for the Assessment and Effect of Different Levels of N- Fertilizers and Spacing on Incidence of False Smut of Rice. Int.J.Curr.Microbiol.App.Sci. 9(10): 2792-2802. doi: https://doi.org/10.20546/ijcmas.2020.910.336 